\title{
Musculoskeletal pain in people with and without type 2 diabetes in Taiwan: a population-based, retrospective cohort study
}

Lee-Wen Pai ${ }^{1,2}$, Chin-Tun Hung ${ }^{3}$, Shu-Fen Li ${ }^{3}$ Li-Li Chen ${ }^{4, *^{*}}$, Yueh- Chin Chung ${ }^{2}$ and Hsin-Li Liu ${ }^{2}$

\begin{abstract}
Background: Musculoskeletal pain in people with type 2 diabetes is a common issue even to this day. The study aimed to explore the 10-year cumulative incidence of musculoskeletal pain, the mean number of doctor visits for musculoskeletal pain, and the mean number of doctor visits for musculoskeletal pain by location in people with type 2 diabetes, compared with respective values for people without diabetes.

Methods: The study utilized a population-based retrospective cohort study design. The subjects were randomly obtained from the Taiwan National Health Insurance Research Database. The diabetic group included 6586 people with type 2 diabetes aged 18-50 years, while the non-diabetic group consisted of 32,930 age- and sex-matched people. Based on the medical records of individuals with musculoskeletal pain in the two groups from 2001 to 2010, the 10-year cumulative incidence of musculoskeletal pain, the mean number of doctor visits for musculoskeletal pain, and the mean number of doctor visits for musculoskeletal pain by location were calculated and compared, with the aim of identifying differences between the two groups.
\end{abstract}

Results: Showed that people in the diabetic group had a higher 10-year cumulative incidence of and a higher mean number of doctor visits for musculoskeletal pain than the non-diabetic group $(p<0.05)$. The relative risk (RR) of the 10-year cumulative incidence of musculoskeletal pain in the two groups was the highest $(R R=1.39)$ for people between 30 and 39 years of age. The mean number of doctor visits for musculoskeletal pain by location was significantly different between the two groups. However, the mean number of doctor visits for limb pain registered the largest difference between the two groups.

Conclusion: People with type 2 diabetes aged 18-50 years had a higher 10-year cumulative incidence of and a higher mean number of doctor visits for musculoskeletal pain than the non-diabetic group. Musculoskeletal pain might directly or indirectly interfere with or decrease the physical activity levels of people with diabetes. Therefore, it is important to detect and treat musculoskeletal pain early in order to promote physical activity and optimize blood sugar control.

Keywords: Type 2 diabetes, Musculoskeletal pain, Incidence

\footnotetext{
* Correspondence: lily@mail.cmu.edu.tw

${ }^{4}$ School of Nursing, China Medical University, No.91 Hsueh-Shih Road,

Taichung 40402, Taiwan

${ }^{5}$ Department of Nursing, China Medical University Hospital, No.91 Hsueh-Shih

Road, Taichung 40402, Taiwan

Full list of author information is available at the end of the article
} 


\section{Background}

Diabetes is one of the leading causes of mortality globally [1]. According to World Health Organization statistics published in 2013, approximately 347 million people worldwide suffer from diabetes, and by 2030 diabetes will be the seventh leading cause of death [2]. In Taiwan, diabetes has remained a serious public health concern ever since 1984, when diabetes rose to the tenth leading causes of death [3].

Type 2 diabetes mellitus is a disease that can lead to a progressive insulin secretory defect on top of insulin resistance [4]. Many studies have indicated that its incidence is related to age [5], obesity [6], family inheritance [7], impaired glucose metabolism [8], and a sedentary lifestyle [9]. People who do not manage their blood glucose levels well often suffer from persistent hyperglycemia, leading to an increase in the production of advanced glycation end products, and causing stiffness of connective tissue and subsequent aggravated musculoskeletal pain [10]. Previous studies have indicated that the causes of increased musculoskeletal pain in people with diabetes are probably related to vascular insufficiency [11], neuropathy [12], decreased insulin-like growth factor 1 [13], accelerated osteoporosis [13], obesity [14], sedentary lifestyle [15] and other factors. Moreover, musculoskeletal pain can directly or indirectly impede or decrease the physical activity levels of people with diabetes. When people with diabetes cannot maintain ideal levels of physical activity, it can affect blood sugar control, causing complications and a decline in quality of life. Therefore, maintaining a proper level of physical activity is a critical part of treatment for people with diabetes, rendering it of equal importance to diet and medicine. Therefore, it is necessary to diagnose and investigate musculoskeletal pain in people with diabetes as early as possible in order to provide proper treatment.

Existing literature on the topic [16-19] indicates a paucity of long-term follow-up research centered on people with type 2 diabetes suffering from musculoskeletal pain. In fact, most of the existing studies used self-report data from patients. Taiwan has enjoyed a complete national health insurance system since its establishment in 1986, and the proportion of the population who are insured is higher than $99 \%$ [20]. The database utilized for this study, the massive and precise Taiwanese National Health Insurance Research Database, was compiled based on diagnostic information from insurance payment records from the National Health Insurance of Taiwan. The objective of this study was to explore the 10-year cumulative incidence of musculoskeletal pain, the mean number of doctor visits for musculoskeletal pain, and the mean number of doctor visits for musculoskeletal pain by location in people with type 2 diabetes aged 18-50 years in Taiwan from 2001 to 2010, and to compare these results with respective values for people without diabetes. This study provides empirical evidence and practical considerations that will allow healthcare professionals to improve the clinical treatment and care for people with type 2 diabetes suffering from musculoskeletal pain. It is important to detect and treat musculoskeletal pain early in order to promote physical activity and optimize blood sugar control.

\section{Methods}

From the Taiwanese National Health Insurance Research Database, one million people were randomly drawn as samples and a 10-year retrospective cohort study was conducted. The subjects included individuals with type 2 diabetes (diabetic group) who were newly diagnosed in 2001 and individuals without type 2 diabetes (non-diabetic group).

The Taiwanese National Health Insurance Research Database recorded the individuals' demographic data and outpatient medical diagnostic codes. First, all people with type 2 diabetes with a Taiwan National Health Insurance expense application diagnostic code 250.0 or A code: A181 (i.e. type 2 diabetes without complications) and who had more than three outpatient records in 2001 were included. Excluded were people having a medical record of diabetes and musculoskeletal pain within 365 days before the first diabetic medical record in 2001. The locations of musculoskeletal pain were broadly categorized as shoulder and neck pain, limb (including arms, hands, and knees) pain, lumbar pain, back pain or pelvis pain, which were referencing categories used by other studies [16]. Musculoskeletal pain in patients with the following Taiwan National Health Insurance payment ICD-9-CM diagnostic codes were included: 719.41 (pain in shoulder joint), 723.1 (cervicalgia), 719.42 (pain in upper arm joint), 719.43 (pain in forearm joint), 719.44 (pain in hand joint), 719.46 (pain in lower leg joint), 719.47 (pain in ankle and foot joint), 729.1 (myalgia and myositis), 729.5 (pain in limb), 719.45 (pain in pelvic region and thigh joint), 724.2 (low back pain), 724.3 (sciatica) and 724.5 (backache). Qualified clinicians classified the patients according to diagnostic codes.

The age range in this study was $18-50$ years, with the upper limit based on the threshold for elderly status being over 50 years in some countries [21]. According to Wilkie et al., who conducted a three- and six-year populationbased prospective study based on 2949 individuals over 50 years of age, the incidence for individuals over 50 years having musculoskeletal pain is related to the healthy aging index. Individuals with a lower index are more likely to have musculoskeletal pain, and the index decreases with increase in age. Therefore, there is a correlation between the health of elderly people over 50 years and the incidence of musculoskeletal pain [22]. With this in mind, and also taking into consideration that widespread pain is a common problem for elderly people and may be influenced by many factors [23], we excluded research subjects 
over the age of 50 to avoid the confounding effects of age and health on the analysis. In addition, the growth and development of children is different from adults, so we excluded children under 18 years old. Therefore based on these criteria, 6586 eligible subjects were selected for the diabetic group.

The inclusion criteria for subjects in the non-diabetic group were: no medical records related to diabetes from 2000 to 2010, no outpatient records related to musculoskeletal pain in 2000, and a record of survival from 2001 to 2010 . Thus 881,968 potentially eligible individuals were identified. After the survival of individuals in both groups in 2010 was confirmed, the individuals were matched according to their age and sex at a ratio of 1:5 (diabetic group: non-diabetic group), and 32,930 individuals were selected for the non-diabetic group.

Next, the use of all categories of outpatient service in both groups was evaluated. Finally, the use of outpatient service for musculoskeletal pain in both groups each year from 2001 to 2010 was examined. The medical records due to musculoskeletal pain from 2001 to 2010 in the two groups according to diagnostic codes were tracked, from which the 10-year cumulative incidence of musculoskeletal pain, the mean number of doctor visits for musculoskeletal pain, and the mean number of doctor visits for musculoskeletal pain by location were calculated. Data from the two groups were then compared to uncover differences.

The 10-year cumulative incidence of musculoskeletal pain was calculated as the number of new patients with musculoskeletal pain from 2001 to 2010 divided by the total number of individuals in the group. The mean number of doctor visits for musculoskeletal pain in each year was calculated as the sum of the doctor visits of every individual due to musculoskeletal pain in each year divided by the total number of individuals in the group. The mean number of doctor visits for musculoskeletal pain from 2001 to 2010 was calculated as the sum of the doctor visits of every individual due to musculoskeletal pain from 2001 to 2010 divided by the total number of individuals in the group multiplied by 10 . The mean number of doctor visits for musculoskeletal pain by location was calculated as the sum of the doctor visits of every individual for each pain location from 2001 to 2010 divided by the total number of individuals in the group multiplied by 10 . Chi-square tests were used to assess differences in the 10-year cumulative incidence of musculoskeletal pain between the two groups. Relative risk (RR) represents the ratio of the 10-year cumulative incidence of musculoskeletal pain in the diabetic group versus the non-diabetic group. Additionally, data for the mean number of doctor visits for musculoskeletal pain and the mean number of doctor visits for musculoskeletal pain by location between the two groups were expressed as mean \pm SD (standard deviation). The significance in differences in the medians of two groups was assessed by the Mann-Whitney $U$ test.

\section{Ethical considerations}

All subject data were encrypted using the same encryption algorithm to cross-link the data while protecting the privacy of the patients. This study protocol was approved by the institutional review board (IRB) of China Medical University Hospital (protocol \#CMUH103-REC1-088).

\section{Results}

This study included 39,516 individuals as subjects: 6586 individuals in the diabetic group and 32,930 individuals in the non-diabetic group. The diabetic group consisted of 338 (5.1\%) individuals from 18-29 years of age, 1338 (20.3\%) from 30-39 years of age, and 4910 (74.6\%) from 40-50 years of age, with 3917 (59.5\%) males and $2669(40.5 \%)$ females. The non-diabetic group consisted of 1690 (5.1\%) individuals from 18-29 years of age, 6690 (20.3\%) from 30-39 years of age, and 24,550 (74.6\%) people from $40-50$ years of age, with 19,585 (59.5\%) males and 13,345 (40.5\%) females. In both diabetic and non-diabetic groups, the 40-50 age group had the highest number of subjects, followed by the 30-39 age group, and the 18-29 age group; the percentage of males was higher than that of females in both groups.

\section{Comparison of the 10-year cumulative incidence of musculoskeletal pain}

The diabetic group had a higher 10-year cumulative incidence of musculoskeletal pain than the non-diabetic group for all age groups and in both females and males $(p<0.05)$, as illustrated in Table 1 . The RR for all age groups and genders was $>1$. Furthermore, the RR was the highest in the 30-39 age group, lowest in the 18-29 age group, and higher in females than in males.

\section{Comparison of the mean number of doctor visits for musculoskeletal pain}

Table 2 shows the mean number of doctor visits for musculoskeletal pain for individuals aged $18-50$ years in the diabetic and non-diabetic groups from 2001 to 2010. Except for 2006, 2007 and 2010, the diabetic group had significantly higher annual and 10-year mean number of doctor visits for musculoskeletal pain than the non-diabetic group $(p<0.05)$.

\section{Comparison of the mean number of doctor visits for musculoskeletal pain by location}

From 2001 to 2010, the mean number of doctor visits for musculoskeletal pain in the neck and shoulders, limbs, lumbar spine, back and pelvis was higher in the diabetic group than the non-diabetic group $(p<0.001)$ 
Table 1 10-year cumulative incidence of musculoskeletal pain and analysis in both groups $(N=39,516)$

\begin{tabular}{|c|c|c|c|c|c|}
\hline & \multicolumn{2}{|c|}{ 10-year cumulative incidence (\%) ${ }^{a}$} & \multirow[t]{2}{*}{$x^{2}$} & \multirow[t]{2}{*}{$\mathrm{RR}^{\mathrm{C}}$} & \multirow[t]{2}{*}{$95 \% \mathrm{Cl}$ of RP } \\
\hline & Diabetic group & Non-diabetic group & & & \\
\hline \multicolumn{6}{|l|}{ Age } \\
\hline $18-29$ & 13.61 & 12.01 & 0.6675 & 1.13 & $1.05-1.22$ \\
\hline 30-39 & 20.18 & 14.51 & $27.3821^{b}$ & 1.39 & $1.31-1.48$ \\
\hline $40-50$ & 23.22 & 19.87 & $28.2924^{b}$ & 1.17 & $1.11-1.23$ \\
\hline \multicolumn{6}{|l|}{ Sex } \\
\hline Male & 19.07 & 16.31 & $17.7761^{b}$ & 1.17 & $1.10-1.24$ \\
\hline Female & 26.56 & 21.40 & $34.2590^{b}$ & 1.24 & $1.18-1.30$ \\
\hline
\end{tabular}

(see Table 3). In relation to the location of musculoskeletal pain, lumbar spine, back and pelvis pain accounted for the highest mean number of doctor visits in both groups. The difference in this value was the highest for limb pain between the two groups.

\section{Utility}

The study found that the 10-year cumulative incidence of musculoskeletal pain and the mean number of doctor visits for musculoskeletal pain were higher in individuals with type 2 diabetes aged 18-50 years than in nondiabetic individuals. Assessing for musculoskeletal pain

Table 2 Comparison of the mean number of doctor visits for musculoskeletal pain in both groups $(N=39,516)$

\begin{tabular}{lllll}
\hline Year & Diabetic group & Non-diabetic group & $Z$ & $p^{c}$ \\
& n: 6586 & $n: 32930$ & & \\
& Mean $^{\mathrm{a}} \pm \mathrm{SD}$ & Mean $^{\mathrm{a}} \pm \mathrm{SD}$ & & \\
\hline 2001 & $0.51 \pm 1.55$ & $0.35 \pm 1.23$ & -3.461 & 0.001 \\
2002 & $0.69 \pm 2.27$ & $0.48 \pm 1.63$ & -2.495 & 0.013 \\
2003 & $0.74 \pm 2.48$ & $0.50 \pm 1.67$ & -2.681 & 0.007 \\
2004 & $0.72 \pm 2.04$ & $0.53 \pm 1.75$ & -2.607 & 0.009 \\
2005 & $0.69 \pm 1.92$ & $0.55 \pm 1.85$ & -2.057 & 0.040 \\
2006 & $0.58 \pm 1.79$ & $0.53 \pm 1.82$ & -0.818 & 0.413 \\
2007 & $0.60 \pm 1.98$ & $0.49 \pm 1.74$ & -1.808 & 0.071 \\
2008 & $0.75 \pm 2.40$ & $0.46 \pm 1.75$ & -3.504 & 0.001 \\
2009 & $0.77 \pm 2.37$ & $0.50 \pm 1.94$ & -1.987 & 0.047 \\
2010 & $0.77 \pm 2.54$ & $0.48 \pm 1.60$ & -1.901 & 0.057 \\
$2001-2010$ & $0.67 \pm 2.12^{\mathrm{b}}$ & $0.48 \pm 1.68^{\mathrm{b}}$ & -7.402 & 0.000 \\
\hline
\end{tabular}

${ }^{\mathrm{a}}$ The mean number of doctor visits for musculoskeletal pain in each year was calculated as the sum of the doctor visits of every individual due to musculoskeletal pain in each year divided by the total number of individuals in the group

${ }^{\mathrm{b}}$ The mean number of doctor visits for musculoskeletal pain from 2001 to 2010 was calculated as the sum of the doctor visits of every individual due to musculoskeletal pain from 2001 to 2010 divided by the total number of individuals in the group multiplied by 10

${ }^{c} p$ value was calculated by Mann-Whitney $U$ test

$S D$ standard deviation in people with type 2 diabetes is necessary, and early detection and intervention might help to maintain ideal physical activity for this population.

\section{Discussion}

The study found that the 10-year cumulative incidence of musculoskeletal pain and the mean number of doctor visits for musculoskeletal pain were higher in individuals with type 2 diabetes aged 18-50 years than in non-diabetic individuals. In addition, the RR for musculoskeletal pain for the diabetic group compared with the non-diabetic group was greater than 1 , which was the highest for individuals in the 30-39 age group. The mean number of doctor visits for musculoskeletal pain in the limbs showed the highest difference between the two groups. The results provide medical professionals information about the incidence of musculoskeletal pain in people with type 2 diabetes. Musculoskeletal pain directly or indirectly interferes with or decreases the physical activity levels of people with diabetes [24]. A systematic assessment of the musculoskeletal system of people with type 2 diabetes should be performed early in the diabetic disease course early and proper detection of musculoskeletal pain [25].

This study found that the mean number of doctor visits for musculoskeletal pain for people with type 2 diabetes in Taiwan within the 10-year period did not increase with the progression of diabetes. This is different from what was observed in a study by Zamani et al., which suggested that the risk of musculoskeletal pain would increase with time over 15 years [17]. This disparity may be because our study assessed the phenomena over a different time span. Additionally, in the years 2006, 2007 and 2010, the mean number of doctor visits for musculoskeletal pain was not significantly different between the diabetic and the nondiabetic groups. This finding might be related to improvement in diabetes treatment and care in Taiwan during this three-year period. Previous studies [26] suggested that persistent hyperglycemia may increase the incidence of 
Table 3 Comparison of the mean number of doctor visits for musculoskeletal pain by location in both groups $(N=39,516)$

\begin{tabular}{|c|c|c|c|c|}
\hline & Diabetic group & Non-diabetic group & Z & $p^{b}$ \\
\hline & n: 6586 & n: 32,930 & & \\
\hline & Mean $^{a} \pm S D$ & Mean $^{a} \pm S D$ & & \\
\hline Neck and shoulders & $0.07 \pm 0.64$ & $0.04 \pm 0.39$ & 2.803 & 0.005 \\
\hline Limbs & $0.27 \pm 0.25$ & $0.17 \pm 0.91$ & 7.405 & 0.000 \\
\hline Lumbar, back and pelvis & $0.35 \pm 1.42$ & $0.28 \pm 1.28$ & 4.157 & 0.000 \\
\hline
\end{tabular}

a The mean number of doctor visits for musculoskeletal pain by location was calculated as the sum of the doctor visits of every individual for each pain location from 2001 to 2010 divided by the total number of individuals in that group multiplied by 10

${ }^{\mathrm{b}} p$ value was calculated by Mann-Whitney $U$ test

SD standard deviation

musculoskeletal pain. During this three-year period, Taiwan had promoted multiple policies on diabetes prevention and treatment [27]. Further assessment is necessary to determine conclusively whether these policies had successfully motivated people with diabetes to follow an active lifestyle and improve their self-management of blood sugar control, which in turn led to a decrease the mean number of doctor visits for musculoskeletal pain.

This study demonstrated that the RR for musculoskeletal pain in people with diabetes was higher for females than males and the 10-year cumulative incidence of musculoskeletal pain for both the diabetic and non-diabetic groups was higher in females than males. This is consistent with findings from other studies [16]. Ramírez-Maestre and Esteve investigated 400 people with chronic spinal pain and their results showed that females had higher pain intensity and pain anxiety than males [28]. Racine et al. reviewed 172 studies from 1998 to 2008 using a systematic literature review, and their results supported the notion that pressure pain thresholds were lower in females than males [29]. The current study analyzed the 10 -year cumulative incidence and the mean number of doctor visits for musculoskeletal pain based on medical records. Further study in the future is needed to elucidate whether the mean number of doctor visits for musculoskeletal pain in females is due to higher pain intensity, higher pain anxiety, and lower pressure pain thresholds among females compared to in males, or if the 10-year cumulative incidence for musculoskeletal pain is indeed higher for females with type 2 diabetes than for males with the same condition. Other possible factors that might influence individuals' motivation for their first doctor visit as well as the mean number of doctor visits for musculoskeletal pain include: age, availability of doctors, and accessibility of medical resources, among others [30]. For this study samples were randomly drawn and individuals were matched according to age and sex to minimize the influence of the other possible factors on the research outcomes.

Among all locations of musculoskeletal pain, the two groups exhibited the highest difference for limb pain. This may be related to long-term poor blood sugar control, which can cause damage in the bones and nerves of the limbs [31,32], and subsequently increase the frequency of musculoskeletal pain in this location. The mean number of doctor visits for lumbar spine, back, or pelvis pains was the highest, which is consistent with the findings of Eivazi [33]. Molsted et al. showed that pain in the lower back is related to high body mass index, sedentary lifestyle and reduced physical function. In addition, pain in the hands, knees, or hip is related to high body mass index [16]. Therefore, high body mass index, sedentary lifestyle, reduced physical function, diseases of the bones and nerves of the limbs, and other factors may contribute to an increase in musculoskeletal pain in the lumbar spine, back, limbs, and pelvis for people with diabetes. Further studies may help address the relative contributions of these related factors.

The power of a study is the largest when the pairing ratio of 1:1 given the sample size is fixed. One way to increase the power of a study is to increase the number of controls when the investigator cannot increase number of cases. In this study, since there were not as many diabetic subjects as non-diabetic subjects, the 1:5 pairing ratio was used to increase the statistical power. This kind of pairing method will not create added bias on the results because each study subject of case and control groups was randomly selected from his/her population. The limitation of this method is that the precision of estimates could only be increased for control group, not for the case group [34]. The age- and sex-matched sampling method eliminated confounding effects of age and sex on the incidence of musculoskeletal pain in the results $[11,22]$. The information was collected from actual medical records to avoid imprecision due to imperfect memory, which is a risk when using self-report data from patients. Since people with diabetes under 18 and over 50 years of age were excluded, the results of this study are limited to people with type 2 diabetes within this age range. Previous studies showed that the causes of an increase in people with diabetes having musculoskeletal pain may relate to hyperglycemia [32], obesity [14], physical functions and other factors [12]. However, because of limitations surrounding access to the National 
Health Insurance Database, related variables, such as blood sugar level, body mass index, weight, or physical functions could not be controlled or adjusted. Therefore, that future research studies should control and adjust for other factors that may cause musculoskeletal pain in order to avoid complications in the interpretation of relative musculoskeletal pain incidence in diabetic versus nondiabetic people in Taiwan.

\section{Conclusion}

The study demonstrates that people with type 2 diabetes aged 18-50 years had a higher 10-year cumulative incidence of musculoskeletal pain and a higher mean number of doctor visits for musculoskeletal pain than those without diabetes. The mean number of doctor visits for musculoskeletal pains by locations was significantly different in both groups. In relation to pain locations, musculoskeletal pain in the lumbar spine, back, and pelvis accounted for the highest mean number of doctor visits for musculoskeletal pain in both groups. However, the mean number of doctor visits for musculoskeletal pain in the limbs registered the largest difference between the two groups. Therefore, professionals should pay special attention to musculoskeletal pain issues when treating people with type 2 diabetes. It is important to detect and treat musculoskeletal pain early in order to promote physical activity and optimize blood sugar control.

\section{Abbreviations}

RR: Relative risk; SD: Standard deviation.

\section{Competing interests}

The authors declare that they have no competing interests.

\section{Authors' contributions}

L.W.P. was responsible for the study concept design, analysis and interpretation of data, drafting of the manuscript, and critical revision of the manuscript for intellectual content. L.L.C. was responsible for the analysis and interpretation of data and critical revision of the manuscript for intellectual content. C.T. H. was responsible for the analysis and interpretation of data and critical revision of the manuscript for intellectual content. S.F. L. was responsible for the analysis and interpretation of data. C.Y.C. and H. L.L. were responsible for the study concept and design. All authors read and approved the final manuscript.

\section{Acknowledgments}

The data from our research came from the Taiwan National Health Insurance Database, and we would like to thank the National Health Research Institution for offering us the data. We also thank the Central Taiwan University of Science and Technology for providing the funding for our research (CTU102-1).

\footnotetext{
Author details

'Department of Public Health, China Medical University, Taichung, Taiwan. ${ }^{2}$ Department of Nursing, Central Taiwan University of Science and Technology, No.666, Buzih RoadBeitun District, Taichung City 40601, Taiwan. ${ }^{3}$ Department of Healthcare Administration, Central Taiwan University of Science and Technology, Taichung, Taiwan. ${ }^{4}$ School of Nursing, China Medical University, No.91 Hsueh-Shih Road, Taichung 40402, Taiwan. ${ }^{5}$ Department of Nursing, China Medical University Hospital, No.91 Hsueh-Shih Road, Taichung 40402, Taiwan.
}

Received: 29 May 2015 Accepted: 16 November 2015

Published online: 20 November 2015

\section{References}

1. Lozano R, Naghavi M, Foreman K, Lim S, Shibuya K, Aboyans V, et al. Global and regional mortality from 235 causes of death for 20 age groups in 1990 and 2010. a systematic analysis for the Global Burden of Disease Study 2010. Lancet. 2012;380:2095-128.

2. World Health Organization. National Diabetes Fact Sheet. Available at http:// www.who.int/mediacentre/factsheets/fs312/en/index.html (2013). Last accessed 4 May 2014.

3. Taiwan Ministry of Health and Welfare. Statistics on the causes of death. Available at http://www.mohw.gov.tw/cht/DOS/Statistic.aspx?f_list_no=312 (2013). Last accessed 2 August 2014.

4. American Diabetes Association. Standards of medical care in diabetes-2010. Diabetes Care. 2010;1:S11-61.

5. Reeves ND, Najafi B, Crews RT, Bowling FL. Aging and type 2 diabetes. consequences for motor control, musculoskeletal function, and whole-body movement. J Aging Res. 2013;doi: 10.1155/2013/508756.

6. Oza-Frank R, Narayan KM. Overweight and diabetes prevalence among US immigrants. Am J Public Health. 2010;100:661-68.

7. Tuchman AM. Diabetes and race: a historical perspective. Am J Public Health. 2011;101:24-33.

8. Wang J, Yuan S, Zhu L, Fu H, Li H, Hu G, et al. Effects of impaired fasting glucose and impaired glucose tolerance on predicting incident type 2 diabetes in a Chinese population with high post-prandial glucose. Diabetes Res Clin Pract. 2004;66:183-91.

9. Cichosz SL, Fleischer J, Hoeyem P, Laugesen E, Poulsen PL, Christiansen JS, et al. Objective measurements of activity patterns in people with newly diagnosed Type 2 diabetes demonstrate a sedentary lifestyle. Diabetic Med. 2013;30:1063-6.

10. Rosenbloom AL, Silverstein $\mathrm{JH}$. Connective tissue and joint disease in diabetes mellitus. Endocrinal Metab Clin North Am. 1996;25:473-83.

11. Browne DL, McCrae FC, Shaw KM. Musculoskeletal disease in diabetes. Practical Diabetes Int. 2001;18:62-4.

12. Arkkila PE, Gautier JF. Musculoskeletal disorders in diabetes mellitus: an update. Best Prac Res Clin Rheumatol. 2003;17:945-70.

13. Sherif EM, Abd Al Aziz MM, Elbarbary NS, Ahmed AM. Insulin-like growth factor-1 in correlation with bone mineral density among Egyptian adolescents with type 1 diabetes mellitus. Int J Diabetes in Dev Ctries. 2011:31:104-12.

14. Esteghamati A, Etemad K, Koohpayehzadeh J, Abbasi M, Meysamie A, Noshad S, et al. Trends in the prevalence of diabetes and impaired fasting glucose in association with obesity in Iran: 2005-2011. Diabetes Res Clin Pract. 2014;103:319-27.

15. Lee PH. Association between Adolescents' Physical Activity and Sedentary Behaviors with Change in BMI and Risk of Type 2 Diabetes. PLoS One. 2014;9, e110732.

16. Molsted S, Tibler J, Snorgaard O. Musculoskeletal pain in patients with type 2 diabetes. Diabetes Res Clin Pract. 2012;96:135-40.

17. Zamani B, Matini SM, Jamali R, Taghadosi M. Frequency of musculoskeletal complications among the diabetic patients referred to Kashan diabetes center during 2009-10. Journal of Kashan University of Medical Sciences. 2011;15:225-31.

18. Slater M, Perruccio AV, Badley EM. Musculoskeletal comorbidities in cardiovascular disease, diabetes and respiratory disease: the impact on activity limitations; a representative population-based study. BMC Public Health. 2011;11:77.

19. Hoff OM, Midthjell K, Zwart JA, Hagen K. The association between diabetes mellitus, glucose, and chronic musculoskeletal complaints: Results from the Nord-Trøndelag Health Study. BMC Musculoskelet Disord. 2008;9:160.

20. Taiwan Ministry of Health and Welfare. National health insurance annual statistical report . Available at http://www.mohw.gov.tw/cht/DOS/Statistic. aspx?f_list_no=312 (2012). Last accessed 2 August 2014.

21. World Health Organization. Definition of an older or elderly person-proposed working definition of an older person in Africa for the MDS Project. Available at http://www.who.int/healthinfo/survey/ageingdefnolder/en. (2014). Last accessed 2 August 2014.

22. Wilkie $\mathrm{R}$, Tajar $\mathrm{A}, \mathrm{McBeth} \mathrm{J}$. The onset of widespread musculoskeletal pain is associated with a decrease in healthy ageing in older people: a populationbased prospective study. PLoS One. 2013:8:1-11.

23. Lekpa FK, Ndongo S, Ka O, Zeba D, Compaoré C, Pouye A, et al. Sociodemographic and clinical profile of chronic pain with neuropathic characteristics in sub-Saharan African elderly. Eur J Pain. 2013;17:939-43. 
24. Stommen NC, Verbunt JA, Gorter SL, Goossens ME. Physical activity and disability among adolescents and young adults with non-specific musculoskeletal pain. Disabil Rehabil. 2012;34:1438-43.

25. van Wilgen P, Beetsma A, Neels H, Roussel N, Nijs J. Physical therapists should integrate illness perceptions in their assessment in patients with chronic musculoskeletal pain; a qualitative analysis. Manual Therapy. 2014;19:229-34.

26. Mathew AJ, Nair JB, Pillai SS. Rheumatic-musculoskeletal manifestations in type 2 diabetes mellitus patients in south India. Int J Rheum Dis. 2011;14:55-60.

27. Ministry of Health and Welfare of Taiwan. Diabetes Prevention and Future in Taiwan. Available at http://www.hpa.gov.tw/English/ClassShow. aspx?No=200803260106 (2014). Last accessed 2 August 2014.

28. Ramírez-Maestre C, Esteve R. The role of sex/gender in the experience of pain: resilience, fear, and acceptance as central variables in the adjustment of men and women with chronic pain. J Pain. 2014;15:608-18.

29. Racine $M$, Tousignant-Laflamme $Y$, Kloda LA, Dion D, Dupuis $G$, Choinière MA Systematic literature review of 10 years of research on sex/gender and experimental pain perception - Part 1: Are there really differences between women and men? Pain. 2012;153:602-18.

30. Yu WC, Fang PL, Ming Y. A customer profile analysis of a regional hospital in southern Taiwan. Yi Bao Yan Jiu Za Zhi. 2006;2:9-18.

31. Deshpande AD, Harris-Hayes M, Schootman M. Epidemiology of diabetes and diabetes-related complications. Phys Ther. 2008;88:1254-64.

32. Wyatt $L H$, Ferrance RJ. The musculoskeletal effects of diabetes mellitus. J Can Chiropr Assoc. 2006:50:43-50.

33. Eivazi M, Abadi L. Low back pain in diabetes mellitus and importance of preventive approach. Health Promot Perspect. 2012;2:80-8.

34. Lin HW, Wang KH, Lin HC, Lin HC. Increased risk of acute myocardial infarction in patients with psoriasis: a 5-year population-based study in Taiwan. J Am Acad Dermatol. 2011;64:495-501.

\section{Submit your next manuscript to BioMed Central and we will help you at every step:}

- We accept pre-submission inquiries

- Our selector tool helps you to find the most relevant journal

- We provide round the clock customer support

- Convenient online submission

- Thorough peer review

- Inclusion in PubMed and all major indexing services

- Maximum visibility for your research 